\title{
LES TECHNOSCIENCES FACE AUX SAVOIRS "POPULAIRES" : MISE EN SENS ET MISE EN SCÈNE DE LA TRANSPLANTATION D'ORGANES
}

\author{
Philippe Chavot ${ }^{1}$, Ulrike Felt et Anne Masseran ${ }^{2}$
}

\section{Introduction}

Depuis le début des années 1990 , le champ des transplantations d'organes traverse en France de grandes difficultés. La pratique de la greffe s'étant développée et consolidée, elle exige un nombre toujours croissant d'organes disponibles. Actuellement, après avoir diminué de façon importante dans les années 1992/1993, le nombre de donneurs stagne : un tiers des personnes en état de mort encéphalique ne peut faire objet d'un prélèvement d'organes à cause du non accord des familles. La solution la plus souvent mise en œuvre pour résoudre cette crise consiste à informer et à sensibiliser "le grand public", en espérant que l'accroissement des connaissances et le rapprochement émotionnel puissent le convaincre et l'amener au don d'organes. L'institution a choisi, dans ce but, de rendre public le fonctionnement

1 Groupe d'Études et de Recherche sur la Science de l'Université Louis PasteurStrasbourg 1 (GERSULP). Les noms des auteurs sont présentés par ordre alphabétique.

2 Institut de Philosophie et de Sociologie des Sciences, Université de Vienne.

Recherches en communication, $\mathrm{n}^{\circ} 15$ (2001). 
des pratiques, celui de l'institution, le vécu de la greffe... en bref, de faire entrer l'idée de la transplantation dans le quotidien. Pourtant, de nombreuses représentations et savoirs permettent de donner des significations -qui ne sont pas forcément issues de la science- au corps, à la vie et à la mort (savoirs populaires, personnels, culturels, religieux...). La technoscience doit ici faire face à toutes ces significations préexistantes.

Comment se déroule cette rencontre ? Tout se passe comme si l'acceptation individuelle et collective de la greffe ne pouvait s'établir qu'au prix d'un abandon -du moins temporaire- des savoirs non scientifiques. On en arrive à une situation où l'adhésion à l'idée du don d'organes est quasi générale sur le plan sociétal mais souvent problématique dans la sphère personnelle. En vue de mettre en évidence certains des éléments constitutifs de cette situation apparemment paradoxale, nous analyserons la mise en scène des représentations relatives à la greffe dans les médias télévisuels. En effet, ces derniers représentent l'un des espaces publics où la médiatisation des savoirs sur le corps s'accompagne souvent d'une "re-formalisation" des significations. La mise en scène médiatique des greffes doit ainsi être comprise comme une mise en sens: des significations plus formelles, souvent scientifiques et standardisées, sont données à la mort, au corps, à l'organe à transplanter ; en outre, ce discours médical dominant s'allie régulièrement à d'autres valeurs plus culturelles (la générosité, la solidarité...) et paraît ainsi se rapprocher de la personne/du citoyen tout en accroissant sa légitimité.

Dans ce contexte, les médias sont tout à la fois tribunes et acteurs. Comment composent-ils avec un savoir scientifique faisant autorité, un ensemble de valeurs qui semblent défendables et avec le "contrat de communication" qui les lie à leurs publics ? En quoi la mise en sens des savoirs qu'ils proposent transforme-t-elle les représentations publiques des sciences et des techniques?

\section{Conflits de savoirs, crises et médiations}

De nombreux proverbes et dictons mettent en évidence l'importance du corps et de ses éléments. Le cœur notamment est pris dans un réseau symbolique particulièrement dense. Ainsi :

- Les yeux sont le miroir de l'âme.

- Quelqu'un d'infiniment bon "a le cour sur la main". 
- La personne volage a un "cœur d'artichaut, une feuille pour tout le monde".

- Et enfin, cette citation de Pascal qui est passée dans notre usage commun des locutions: "Le cour a ses raisons que la raison ne connaît point".

Dans l'espace médiatique, ce type de discours existe, certes, mais il apparaît la plupart du temps comme secondaire, voire même parfois comme non-souhaitable. Depuis les années 1970 (c'est-à-dire le moment où la médiatisation des greffes d'organes s'est intensifiée), le discours scientifique et technologique tend à s'imposer comme l'ultime référence pour expliquer le corps ${ }^{1}$. Par rapport à la force des images et des discours technoscientifiques, le premier champ de significations semble s'affaiblir au point d'être parfois littéralement ignoré. Les trente années de la médiatisation des greffes sont ainsi marquées par cette association des significations du corps aux valeurs technoscientifiques, comme ces exemples peuvent le suggérer ${ }^{2}$ :

- "Nous ce qui nous intéresse ce sont des personnes qui ont des organes qui soient bons pour la réussite de la greffe, donc ça s'échelonne, si vous voulez, entre 15 ans, 18 ans et 50 ans. (...) il y en aura à peu près $10 \%(\ldots)$ qui seront des donneurs éventuels, c'est-àdire qui seront morts dans un hôpital, qu'on aura pu mettre sous une machine pour conserver leur corps à fin de prise d'organes" 3 .

- "Un cour artificiel avait été alors posé (...). Mais depuis quelques jours son état [du patient] était devenu de plus en plus préoccupant (...).Toutes ces complications ont entraîné la mort, le cœur artificiel a, lui, parfaitement fonctionné. Pour le professeur Cabrol, cet échec

1 On note même, dans les années 1980, une quasi-hégémonie du discours scientifique, reléguant à l'arrière-plan tout autre mode de compréhension du corps. Cette évolution tient, d'une part, aux progrès de la greffe, notamment en matière d'immunologie et de réponses médicamenteuses, et par voie de conséquence à sa prescription pour un nombre croissant de pathologies. D'autre part, la personnalité du prof. Cabrol, directeur de France-Transplant et promoteur-vedette de la greffe dans les médias, joue un rôle important. En effet, il parle en qualité de pionnier et de chirurgien cardiaque. Cette hégémonie des explications scientifiques du corps s'atténuera (tout en restant dominante) au cours des années 1990 avec la création de l'Établissement Français des Greffes (EFG), les discussions autour des lois bioéthiques, la présence de nouveaux acteurs sur la scène médiatique...

2 Ces exemples sont tirés de notre étude sur la médiatisation de la greffe : cf. P. Chavot, U. FElT, A. MASSERAN, "Entre savoirs et confiance : médiation et crise de la transplantation en France", rapport final présenté à l'Établissement Français des Greffes (convention 98/914/17/575(99)), Strasbourg/Vienne, juillet 2001.

3 Prof. Kuss, intervenant dans l'émission Aujourd'hui Madame, deuxième chaîne, 212-1976. 
ne remet pas en cause la poursuite de son programme de transplantation, mais il envisage de sélectionner plus sévèrement les malades qui, à l'avenir, pourraient bénéficier d'un cœur artificiel"'.

- "Accident de circulation. Premiers secours. Transfert en service de réanimation. Le cerveau n'est plus irrigué, il se détruit en 4 minutes, c'est la mort cérébrale. Plus d'espoir, c'est des machines qui le [la personne accidentée] maintiennent en vie"'2.

- "Tout ce que nous sommes capables de réparer a un correspondant en physique et en chimie. (...) tout ce qu'on va réparer c'est la machine humaine"3.

- "Depuis plus de 5 mois, en attente d'un greffon, André et sa machine ne font plus qu'un. Branchée en permanence, elle relaie son cœur défaillant"4.

Il existe certes des formes intermédiaires entre ces deux formes de communication et champ de significations, mais elles sont rarement présentes sur la scène médiatique. Dans les entreprises de communication les plus récentes, celles menées notamment par l'EFG, on observe une volonté de faire coexister les significations plus quotidiennes du corps et le savoir formel technoscientifique. Mais, cette rencontre semble difficile : en effet, on observe l'émergence d'un discours à deux têtes dont les deux dimensions ne s'intègrent pratiquement jamais l'une à l'autre. Cette rupture n'est pas sans conséquences, et nous faisons l'hypothèse ici qu'elle est au centre de ce que, au courant des années 1990, les acteurs/promoteurs de la greffe ont appelé, en France, une "crise de confiance".

\subsection{Une crise de confiance ?}

Lorsqu'il est fait référence à une notion aussi radicale que celle de crise, il faut être conscient de ce qu'elle présuppose. Une crise ne peut, par définition, surgir qu'en rupture avec une situation normale.

1 Commentaire en voix off, Lucie Soboul, "Mort transplanté Cabrol", JT Midi, Antenne 2, 24-04-1986, 13 h03.

2 Commentaire en voix off, "Enquête dons d'organes", JT 20h, France 2, 8-04-1997, $20 \mathrm{~h} 23$.

3 Prof. Jean-François Mattéi, interviewé au cours de l'émission De quoi j'me mêle? $\mathrm{n}^{\circ} 57$, consacrée au thème, "Médecine : a-t-on le droit de tout faire", Arte, 15-06$2000,20 \mathrm{~h} 45$.

4 Commentaire en voix off, "Le manque de dons d'organe en France", JT 20h, TF1, 02-10-2000, 20h24. 
Aussi, il faut se demander quel est le sens, et quelles sont les implications de cette "normalité".

Lorsqu' aucun dysfonctionnement ne vient les remettre en cause, les relations complexes entre science et société sont symboliquement enfermées dans des "boîtes noires". On sous-entend leur efficacité et leur simplicité. Elles restent ainsi invisibles et non questionnées. Les publics concernés semblent se satisfaire des "faits" et ne demandent guère à participer à ces processus. La science est largement perçue comme une instance productrice de certitudes, et c'est précisément la distance qui nous sépare de la production des connaissances scientifiques et de leurs applications qui confère aux résultats scientifiques une certaine robustesse. Harry Collins résume ce phénomène par une phrase : "La distance crée l'enchantement".

Par conséquent, on ne manque pas d'être étonné lorsque les publics -où plus précisément des publics spécifiques- résistent aux transformations technoscientifiques. C'est en situation de crise ou de controverse que la boîte noire est ouverte, lorsque les règles jusque-là admises ne peuvent plus faire valoir leur pouvoir de manière illimitée. Mais il convient de se demander pour qui il y a crise et quels sont les sens qui lui sont conférés.

La crise qui bouleverse le champ de la transplantation depuis le début des années 1990 est d'abord définie arithmétiquement : le nombre de prélèvements d'organes est trop faible au regard de la demande, faiblesse tenant pour une large part à l'attitude des familles, refusant "trop souvent" le prélèvement sur un proche. Mais ce lien de cause à effet peut être interprété très différemment suivant les "mondes sociaux" dans lesquels les différents acteurs sont engagés ${ }^{2}$.

Il y a "crise" en effet, et une crise douloureuse pour les patients et leur famille qui attendent, qui ont parfois le sentiment que la solidarité sociale ne se concrétise pas dans le don. Il y a crise pour les acteurs mobilisés professionnellement dans le domaine de la

$1 \mathrm{H}$. Collins, "Certainty and the public understanding of science on television", Social Studies of Science, vol. 17, 1987, p. 692.

2 Nous reprenons ici la définition de Karin Garrety: les mondes sociaux correspondent à des unités plus ou moins structurées dans lesquelles les personnes partagent des ressources ou des informations qui impliquent des hypothèses communes sur ce qui est important et sur ce qui doit être fait. Chaque personne participe à plusieurs mondes sociaux, organisés entre le travail, la famille, la politique, la religion. Cf. K. GarReTY, "Social Worlds, Actor-Networks and Controversy: The case of Cholesterol, Dietary Fat and Heart Diseases", Social Studies of Science, vol. 17, 1997, pp. 730-731. 
transplantation (institutionnels, médecins, décideurs, mais aussi les médias...). Le manque d'organes menace ici une pratique, liée aux technologies médicales de pointe, au prestige de la profession de chirurgien, à l'image de l'institution hospitalière. Cette crise met donc en danger tout un monde dans lequel des investissements lourds et d'ordres divers ont été placés.

Selon ces professionnels, la responsabilité en incomberait en partie au sensationnalisme médiatique qui a monté en épingle des "rumeurs" concernant les trafics d'organes et qui donne une image négative de l'institution. Coupables tout désignés également certains gestionnaires d'associations médicales, mais également les institutions hospitalières, qui n'ont pas su éviter certaines dérives... ${ }^{1}$. Enfin, les nouvelles dispositions légales qui se mettent en place au début des années 1990, imposant aux médecins de recueillir le témoignage des proches, ont parfois été accusées de nourrir la pénurie d'organes ${ }^{2}$.

Mais une question majeure reste ouverte : y a-t-il réellement crise pour l'ensemble de la société ? L'hypothèse d'une crise de confiance présuppose que les donneurs potentiels se soient transformés en porteurs du refus. Dans ce cadre, la solution à la crise passerait obligatoirement par l'acceptation publique, la plus large possible, de l'idée qui veut que la greffe soit "l'affaire de tous". Nous assistons là à une entreprise de formalisation de la crise : elle devrait avoir une signification acceptable et appropriable par l'ensemble de la population.

Dans ce discours, le cœur du problème n'est pas la transplantation per se mais le degré de confíance que les publics accordent à l'institution et à la pratique de la transplantation, toutes deux mises à mal par les médias. Toutefois, peut-on raisonnablement penser que les publics soient à ce point soumis à la "dictature" des médias ? En fait, l'hypothèse de la "crise de confiance" s'appuie sur un postulat qui n'a jamais été remis en cause : il serait "normal" de donner ses organes. Mais donner ses organes ne paraît normal que selon certaines perspectives (de la science, de l'institution, du droit...). Repensons à

1 Ces dérives deviennent visibles sur la scène médiatique à partir de la fin des années 1980. Il s'agit, par exemple, de réseaux organisés permettant à des patients italiens de bénéficier de greffons français, d'enrichissements personnels, d'abus au niveau des prélèvements d'organes...

2 Pour parer à la crise, une institution d'État, l'Établissement Français des Greffes, a été créée en 1994, dans le cadre des lois dites bioéthiques. Elle est chargée de réguler le champ de la transplantation, les processus médicaux et sociétaux entourant le prélèvement et la transplantation d'organes, mais aussi d'informer. 
nos proverbes et dictons : s'il y a crise, c'est également une crise de la "mise en sens" du corps et de la science.

\subsection{Les médias alliés de la science?}

Si la greffe doit devenir "l'affaire de tous", les médias représentent un lieu stratégique. La vulgarisation des connaissances en matière d'immunité, de pathologies, de guérison et d'interventions techniques sur le corps parvient dans tous les foyers à travers les journaux et surtout le petit écran. Les médias sont le premier lieu d'intégration des idées scientifiques dans la société. Ils permettent que se constitue un savoir commun, indispensable à la compréhension publique et, plus encore, à la légitimation de la transplantation.

Les entreprises de communication sur les greffes, alliant actuellement médias et acteurs de la greffe, visent à mieux informer sur tous les aspects du domaine, des modalités de prélèvement au transport de l'organe, des maux qu'il s'agit de guérir jusqu'aux effets bénéfiques de la greffe. En ce sens, elles véhiculent l'idée suivant laquelle le public ne serait réticent que parce qu'il souffre d'un manque de connaissances, notamment scientifiques : l'acquisition d'un tel savoir le conduira à entendre raison, donc à accepter le développement du champ, et surtout à le soutenir en acceptant de donner ses organes.

Pourtant, la communication prend place dans des structures complexes qui se recouvrent souvent. Aussi, la médiatisation des sciences n'est, de ce fait, pas un lieu où le savoir est transmis de manière linéaire depuis la science vers les publics. C'est également un lieu dans lequel les interprétations émanant des différents acteurs sont négociées. Michel Cloître et Terry Shinn mettent bien ce point en évidence :

Dans le cas de la popularisation de la science, le langage, le raisonnement et les images du propos ne parviennent pas à élucider le phénomène mais, au contraire, ils tendent à générer une incompréhension conceptuelle. La popularisation ne constitue donc pas un instrument efficace pour la transmission d'une meilleure connaissance du monde physique. Sa force et sa pertinence reposent sur les liens qu'elle établit entre un sujet scientifique et la sphère sociale ${ }^{1}$.

1 M. CloÎTRE et T. SHINN, "Enclavement et diffusion du savoir", Information sur les Sciences Sociales, vol. 25, 1986, pp. 163. 
Bien que les modes techniques de production des médias classiques, comme la presse ou la télévision, rendent d'emblée impossible une interaction directe avec les publics, ces médias doivent surtout être compris comme des espaces de communication interactifs. D'un côté, en effet, les publics accèdent d'une certaine manière à la scène, à travers le journaliste qui les représente et se les représente. Ils exercent ainsi une influence sur ce qui est écrit ou montré, mais aussi sur la manière dont on écrit ou montre. D'un autre côté, les informations proposées par les médias sont interprétées et intégrées dans les contextes de savoirs et d'expériences propres aux lecteurs ou aux téléspectateurs. Dans les deux cas, ces informations sont soumises à des processus de négociation.

Or, les entreprises de communication concernant la transplantation se caractérisent par un remarquable consensus entre scientifiques, politiques et médias : porté par une information scientifique que l'on imagine être convaincante, le don d'organes acquiert une représentation unidimensionnelle. Il est souvent présenté comme un dû. On doit donner au nom des valeurs associées à la médecine, bonnes et justes ; on doit donner au nom de la générosité et de la civilité, valeurs également universelles. Mais ces valeurs, justement parce qu'elles prétendent pouvoir être admises par la collectivité, seront-elles vraiment acceptées alors qu'elles présupposent l'abandon de tout un ensemble d'autres savoirs, d'autres perceptions du corps, d'autres valeurs?

Par ailleurs, bien sûr, la question reste ouverte de savoir jusqu'à quel degré la relation entre médias et publics est réellement contrôlable par les scientifiques, ou par d'autres institutions. De plus, il est évident que la manière dont les publics négocient et réinterprètent l'information est loin d'être maîtrisable -et heureusement. Si les médias s'allient aux sciences, ce processus ne peut donc être que partiel et doit toujours être requestionné.

\section{Confrontations et domestications des savoirs}

Ici émerge une nouvelle problématique. Depuis les années 1970, et plus encore dans les décennies suivantes lorsque le champ connaît un véritable essor, il semble admis que ce sont les savoirs ou les 
perceptions non scientifiques qui constituent le dernier obstacle aux développements et au progrès du champ.

Dans les entreprises de sensibilisation, tout un ensemble de frontières est tracé pour conférer à la définition scientifique du corps une valeur dominante. Tout d'abord, il s'agit de faire reconnaître la science en tant qu'instance de progrès humain (elle sauve des vies), ensuite, d'expliquer les concepts -comme celui de mort encéphalique- et enfin de réaliser un lien avec tout un ensemble de valeurs citoyennes -solidarité, générosité, bienfaisance... Cette frontière vise à montrer ce qu'est la médecine, à quoi elle sert, mais aussi ce qui nuit à son développement -irrationalité due à la douleur, croyances erronées, savoirs populaires... Tout se passe comme si, derrière la volonté de faire reconnaître publiquement cette frontière, se cachait une crainte : les "autres savoirs" sont considérés comme des "menaces" pour la science.

Par exemple, la douleur de la mère face au décès de son enfant est souvent invoquée comme l'une des causes principales de l'opposition au prélèvement. C'est bien, dans ce cas, deux perceptions -associées à deux types de savoirs- qui s'affrontent : la mère voit son enfant comme un tout, incarnant la vie, l'amour, toute une vie d'affection; la médecine voit un assemblage d'organes, chacun susceptible de sauver une vie. Dans ce contexte, la douleur sera formalisée : la douleur est rejetée dans l'irrationalité qui est classiquement représentée comme une entrave au progrès, à la bonne marche de la médecine et de la société.

Il faut se demander ici sur quoi reposent ces craintes et où réside le pouvoir des "savoirs populaires". Analysant ce type de processus d'autonomisation de la science, R.G.A Dolby explique que, très souvent,

Le savoir populaire, duquel l'establishment scientifique tente de se dissocier, a un style qui est plus adapté aux espaces publics que celui de la science orthodoxe. Il est relativement large et propose des observations et des explications concernant de nombreuses questions "populaires". Il est visionnaire et programmatique plutôt que rigoureux et testable. Et il suscite l'adhésion sans l'entremise d'un expert scientifique'.

1 R.G.A. DOLBY, "On the autonomy of pure science. The construction and maintenance of barriers between scientific establishments and popular culture", in Scientific Establishments and Hierarchies, Sociology of the Sciences, vol. 6, 1982, pp. 272. 
Le domaine de la médecine est particulièrement problématique dans cette perspective, car il est -presque traditionnellementparcouru par des discours antiscientifiques ou alternatifs. Les profanes ont des connaissances et des représentations de leur propre corps, ce qui les conduit à élaborer des systèmes de savoir complexes. Le fonctionnement du corps est d'abord ressenti, ce qui amène chacun à décider de ce qui est bon ou mauvais pour lui. Aussi, lorsque les médias présentent une image hautement technologisée de certains domaines extrêmement chargés de significations et de symboliques culturelles, comme la mort, on ne les reconnaît pas, voire même on ne les accepte pas.

Nous avons choisi trois types de relations mobilisant à la fois le savoir médical et les "autres formes de savoir" pour mettre ces problématiques en évidence : le rapport au corps, le rapport au fonctionnement du corps, et le rapport à la mort.

\subsection{Le rapport au corps}

Nous percevons le corps dans lequel nous vivons comme un organisme total, entier. Le corps est un ensemble à travers lequel nous faisons les expériences les plus intimes. Et d'ailleurs, d'un point de vue immédiat, le corps et le psychisme sont rarement perçus comme deux choses séparées : ce que nous ressentons à travers notre corps, nous le ressentons aussi psychiquement (une blessure cause de la douleur et de la tristesse...).

Face à ce vécu, la transplantation semble proposer de "réparer" la machine, non pas en tenant compte du tout mais en sélectionnant et en remplaçant la pièce défectueuse par une autre pièce analogue. La mise en scène médiatique des transplantations rend compte de cette réalisation à travers des éléments récurrents. Ainsi, la fameuse "glacière" destinée au transfert de l'organe d'un point à un autre est régulièrement mise en scène ${ }^{1}$. On insiste également sur le soin particulier avec lequel les protagonistes de la greffe manipulent l'organe à transplanter : tout un ensemble d'actions s'organise autour de lui, il est au centre de la scénarisation. En revanche, l'aide à la survie du

1 Le fait qu'elle ressemble à une glacière de "pique-nique" n'est certainement pas étranger à la constitution d'un champ de signification. En effet, cette ressemblance semble "réduire" la distance entre la pratique de la greffe et le monde familier et quotidien. 
corps qui le contient (le traitement très lourd qui suit la transplantation) n'apparaît pratiquement pas. Tout se passe comme si ces organes pouvaient fonctionner de façon indépendante.

Autre exemple, les médias ne présentent pas un corps, susceptible d'être malade ou sain, ou dans un état intermédiaire, mais deux corps qui s'opposent et dans lesquels les publics ne peuvent se reconnaître. D'un côté le corps malade, défini par la maladie : il ne s'agit plus d'une personne, mais de "quelqu'un" dont le fonctionnement est soumis à la technique médicale, dont la "vie" dépend de tuyaux, de sondes et de machines omniprésentes. De l'autre côté, le corps "réparé".

Int. Charles : Cette greffe, je l'attend... depuis le premier jour de mon insuffisance rénale. J'y ai rêvé. J'espérais qu'elle arriverait dans un délai relativement court. (...) je suis toujours en attente de transplantation, quoi, en fait, sept ans à vivre au rythme des dialyses.

Voix off : Tous les jours, Charles doit rester 10h branché à une machine (...)
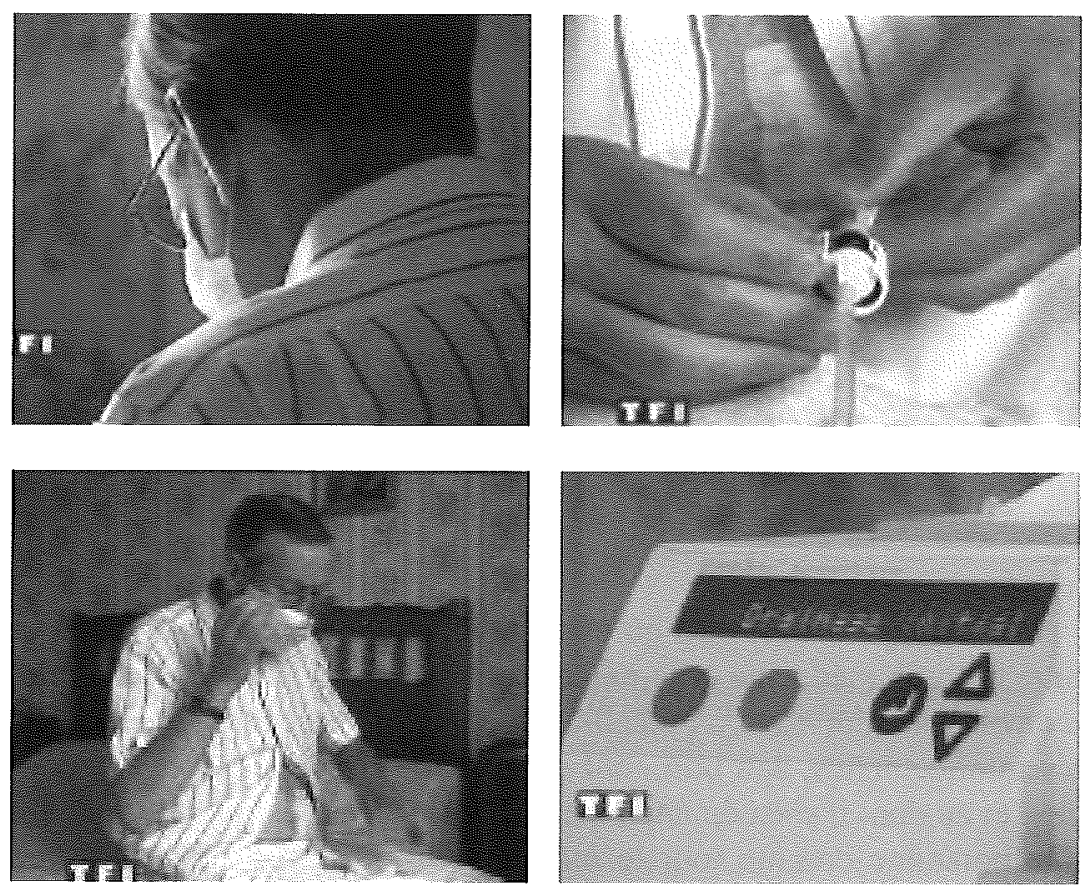
Voix off : Gilles a eu plus de chance. Il a reçu un rein en 1995. Après 4 ans de dialyse, il a vécu sa transplantation comme une renaissance.

Int. Gilles: J'ai été épaté de voir qu'on récupérait à une vitesse extraordinaire et qu'après quelques jours, je pouvais marcher, manger normalement. Aujourd'hui, je travaille comme un damné, ..., je fais tous les sports possibles et imaginables, je vis tout à fait comme tout le monde.

Voix off : Gilles peut de nouveau écrire des romans, mais aussi savourer les plaisirs du quotidien... Un rêve pour Charles.
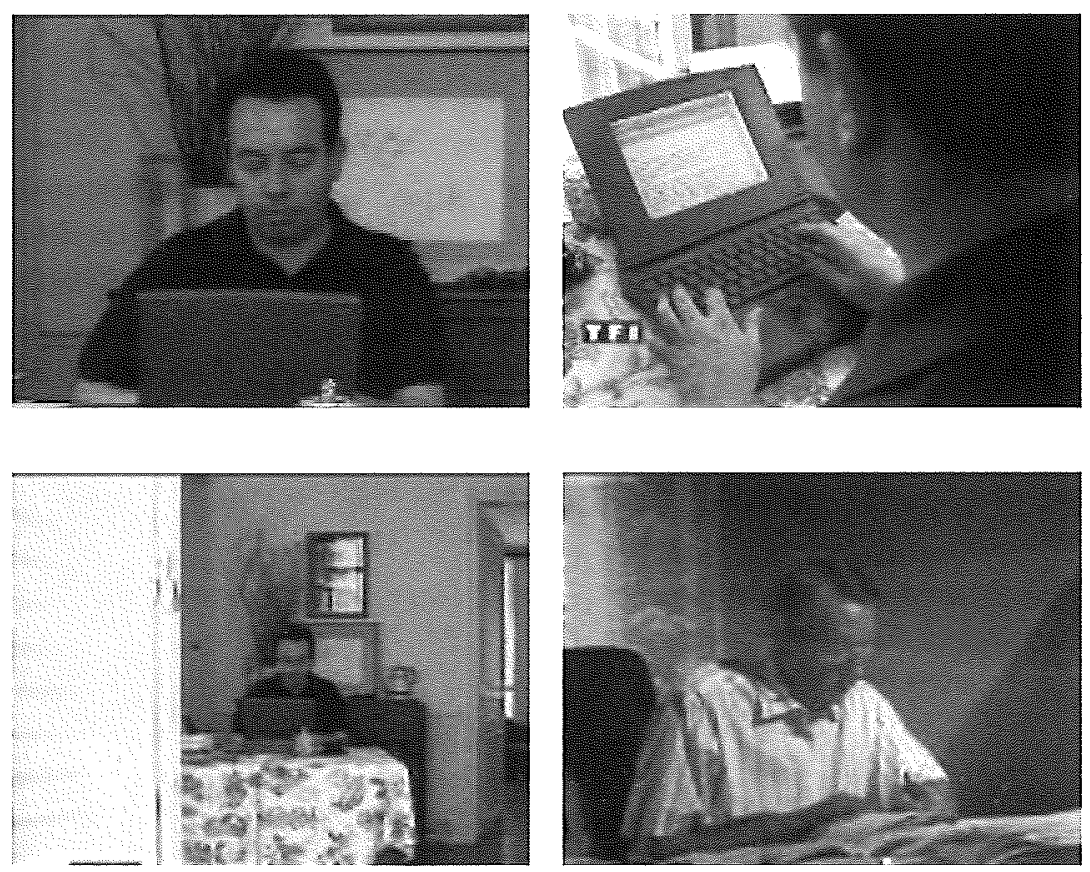

Illustration 1 : Dépendance et libération.

("Don d'organes", JT 20h, TF1, 16-05-1998, 20h28)

L'être qui n'était plus qu'un patient entièrement déterminé par la lutte entre la médecine et le mal et par sa dépendance à la technique, redevient un individu : on focalise sur ses exploits sportifs, ses capacités intellectuelles, son bon goût culinaire... Grâce à la technomédecine et au don, il s'est émancipé du mal. Mais ce n'est pas pour autant une personne comme tout le monde : le "corps réparé" est animé d'une vie extrêmement intense. 
Interview d'Idaline, greffée du cour : La première fois que j'ai fait $d u$ sport, je me suis levée d'un coup, j'ai entendu mon caur battre, normalement, sans un essoufflement. Euh, oh vraiment j'étais très émue, j'ai versé une larme, je me suis dit c'est pas possible, j'ai retrouvé la vie normale c'est formidable.

C'est beau de pouvoir donner ses organes pour que quelqu'un puisse continuer à vivre, je remercie vraiment, vraiment les gens qui disent oui. C'est grâce à ça que je suis là.
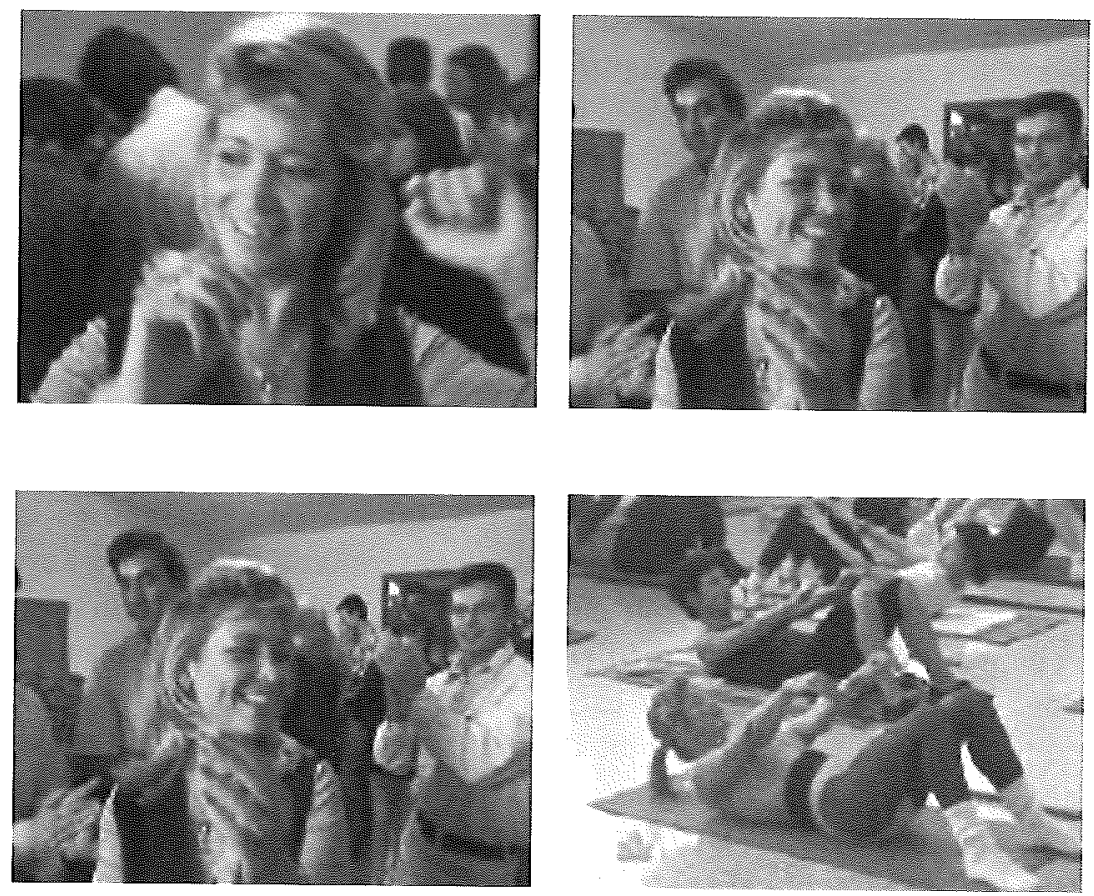

Illustration 2 : La greffe, ça marche.

(Dominique GIULIANI, "Greffes : état d'urgence", Savoir plus santé, France 2, 16-11-96, 13h48)

Tout se passe comme si cet engagement dans de nouvelles règles de vie s'apparentait à une éternelle reconnaissance envers la médecine et envers "l'étranger" qui est en lui. En fait, ces deux représentations du corps se lisent comme deux histoires qui n'ont pas grand-chose à voir avec le rapport privilégié que nous avons à notre corps, elles vont même jusqu'à contredire le vécu quotidien de la plupart des personnes 
qui n'ont pas de problèmes de santé "particuliers" et qui ne se livrent pas pour autant à de tels exercices.

\subsection{Le fonctionnement du corps}

Cette diversité des approches et des définitions est également perceptible à travers les métaphores qui entourent le corps et certains organes. La logique technomédicale s'exprime à travers un ensemble d'images et d'éléments de signification référant à une conception du corps qui prennent un sens très précis lorsqu'il s'agit de transplantation : considérant le corps comme une machine faite de rouages interchangeables, la transplantation est la réalisation de la métaphore du corps-machine. C'est sur ce principe, accompagné des valeurs de solidarité et de générosité, que se fonde l'idée du don d'organes : nous sommes "mécaniquement" tous similaires et sensés être unis par les mêmes valeurs sociales, ce qui fait de nous tous de potentiels receveurs et donneurs d'organes.

Cette conception pose problème à deux niveaux (au moins). Tout d'abord, il suffit de se rendre compte du nombre de dictons et proverbes référant au cœur ou aux yeux par rapport à d'autres organes pour s'apercevoir qu'ils sont culturellement chargés de valeurs qui échappent à la définition médicale largement diffusée et vulgarisée dans nos sociétés : "le cour est un muscle qui pompe". De plus, le cœur, notamment, fait l'objet de nombreuses métaphores. Or ces dernières nous donnent parfois la possibilité d'exprimer les choses les plus profondes, celles auxquelles on attache souvent le plus d'importance. Elles structurent et soutiennent notre manière d'appréhender et d'exprimer le monde, ne sont pas innocentes et elles témoignent de valeurs implicites que nous associons aux choses. Cette importance sociale, culturelle, personnelle est abolie lorsqu'on parle en termes de corps-machine.

Ensuite, parallèlement à la mise en scène qui promeut le don d'organes, les médias font exister un discours sur le corps qui accentue son individualité et qui le valorise en tant que bien propre à la personne : la publicité, les top models, les sportifs, le désir de vie saine... Le corps d'aujourd'hui est un corps qui se modèle, se soigne, sur lequel on travaille : on lui fait du bien et il nous fait du bien, le corps représente l'individu. Il est difficile de l'imaginer morcelé et distribué à d'autres, surtout lorsqu'il a fait l'objet d'efforts qui lui 
donnent son apparence jeune, tonique, saine. Les normes actuelles (esthétiques et sanitaires) entourant le corps constituent très certainement un obstacle au don d'organes : le corps -ou plutôt ce qu'on a réussi à en faire- devient actuellement particulièrement précieux. De plus, cette construction du corps privé permet aussi à l'individu de se forger un savoir sur son fonctionnement, savoir qui n'est pas forcément compatible avec le savoir commun qui devrait légitimer socialement la greffe'.

\subsection{Le rapport à la mort}

L'état de mort encéphalique est la condition sine qua non à laquelle la greffe est possible. Or cette mort du cerveau représente une véritable redéfinition de la limite à partir de laquelle on décide qu'un individu n'a plus d'avenir ni de présence dans ce monde. Comment faire accepter une définition technique de la mort qui remet en cause le savoir immédiat de "ce que l'on voit", de ce que l'on espère ou de ce que l'on "sait" sur la mort ? En effet, lorsqu'on fait appel à leur témoignage, les familles doivent d'abord se convaincre de la mort du proche qui est devant eux, rose et respirant, pour consentir au prélèvement. Cette acceptation est d'autant plus difficile que la mort encéphalique implique une remise en cause du sens commun que la médecine elle-même a contribué à établir. Très longtemps, l'idée dominait que la mort se décelait à travers l'arrêt de la respiration et du cœur. Or, avec la notion de mort encéphalique c'est le lieu même du constat de mort qui se modifie : le cerveau devient le siège de la vie, et le cœur n'est plus que subalterne.

Les écrans affichent un encéphalogramme plat, des clichés d'artériographie montrent que le sang ne circule plus. Mais ces signes sont produits par des machines, et non directement par le corps qui semble, lui, être en vie. La visibilisation, l'objectivation technique de la mort entre alors encore plus en conflit avec les savoirs personnels.

1 Cela pose un problème d'acceptation : l'attitude normative vis-à-vis du corps individuel est difficilement compatible avec l'idée que certains individus seraient "hors-normes" (fumeurs, alcooliques) et pourraient bénéficier du "précieux" organe. Plus profondément, comment les médecins gèrent-ils la frontière entre l'univers de la maladie -le "royaume de la maladie" comme l'appelle Susan Sonntag - et le monde des personnes saines, répondant à d'autres logiques, d'autres normes ? (S. SonNTAG, La maladie comme métaphore. Le sida et ses métamorphoses, Paris, Christian Bourgois, 1993). 
Nous n'avons pas l'habitude de faire signifier la mort à travers des signes inscrits sur des écrans ou des photos mais à travers des signes beaucoup moins médiatisés, à travers une perception directe du corps. La visibilisation médicale de la mort exige donc une confiance dans la technique, une acceptation de ce qui est dit "objectif" (la machine), et surtout la conviction que l'objectivité médicale est supérieure à la subjectivité personnelle.

Voix off : Au-delà de la mort, il y a parfois la vie...
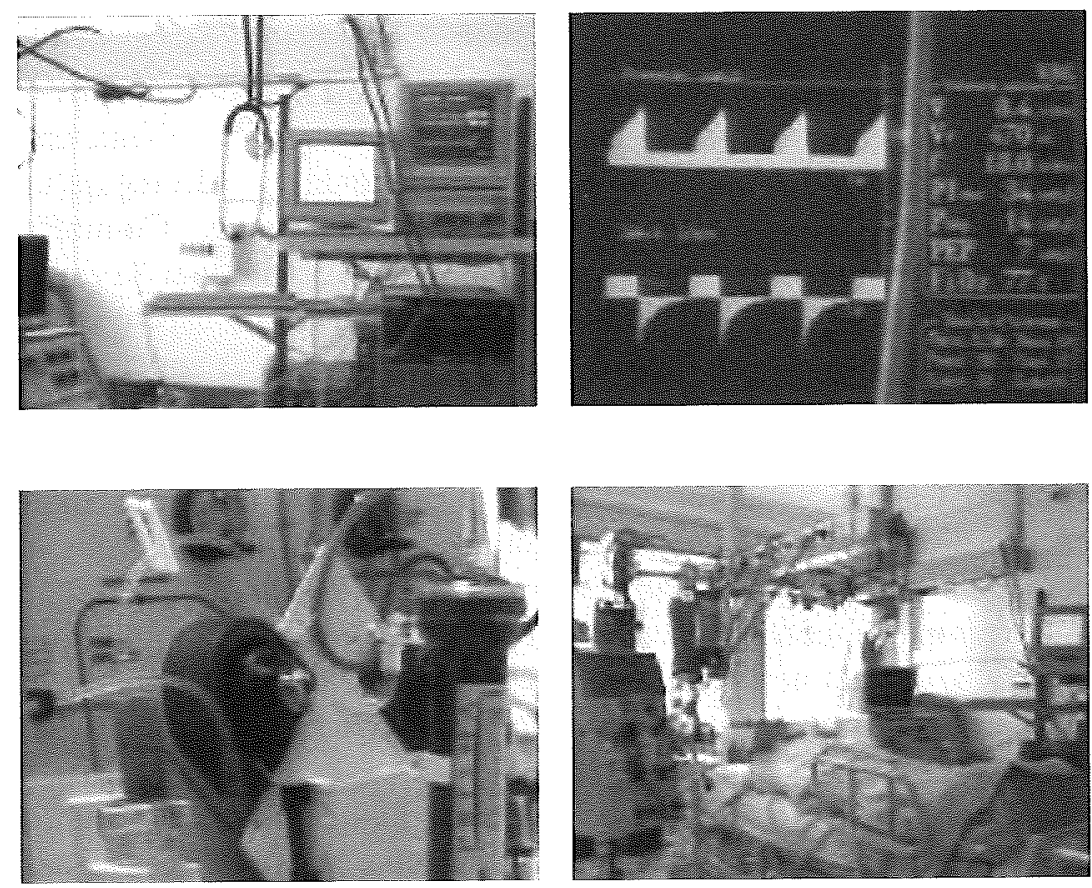

Illustration 3 : Images technicistes de la mort

(“Campagne dons d'organes", JT 20h, France 2, 07-07-1998, 20h09)

En outre, la définition de la mort fondée sur l'artifice technique est quasiment imposée. Comment, dès lors, établir cette relation de confiance entre les familles qui sont appelées à accepter le prélèvement et les médiateurs ? En effet, cette communication est unilatérale, les membres de la profession médicale diffusent leur savoir sans tenir compte des savoirs des familles. Or, la confiance n'est pas donnée, il 
s'agit d'une relation qui se construit grâce à l'activité et à la participation de tous les partenaires. On peut dire, pour paraphraser Antony Giddens, que la confiance doit se construire, ce qui présuppose en règle générale, qu'on échange avec l'autre, que l'on se montre émotionnellement ouvert à lui'. Pourtant, dans le cas qui nous intéresse, les savoirs "autres" sur la mort ne sont guère pris en compte, ils sont souvent considérés comme négligeables.

Par ailleurs, accepter de donner le corps d'un proche revient aussi à faire sien des régimes de signification, des règles de vie et de mort propres à un monde hospitalier qui est largement étranger à la plupart d'entre-nous. Il faut alors pouvoir se soumettre au discours et au pouvoir d'un interprète -le médecin- qui parle une langue inhabituelle. Cela implique, une fois encore, une relation de confiance. Le contexte de la médiation joue un rôle important dans le processus de négociation personnelle qui doit amener le proche d'une personne en situation de mort encéphalique à donner ou non ses organes.

\section{Deux fictions antagonistes}

Ces confrontations du savoir formel des technosciences, fort de sa mise en sens médiatique, et des "autres" savoirs moins formalisés, amènent à différents types de réactions.

L'absence de dialogue social et juridique concernant la pratique et la logique de la transplantation offre un terrain propice à l'émergence de contre-discours. Ainsi, en 1978, l'intrigue du film Coma de Michael Crichton assimile le prélèvement d'organes à un vol, perpétré par des médecins assassins. Et cette vision trouve progressivement un lieu d'expression dans l'espace public à partir de la fin des années 1980. De nombreux articles et reportages sont consacrés aux trafics d'organes. Même s'il ne s'agit que de rumeurs, comme tente de le démontrer Campion-Vincent, il faut se demander si, au fond, l'idée de trafic ne correspondrait pas à une représentation latente de la greffe et des pratiques qui l'entourent ${ }^{2}$. L'opération est assimilée à un "vol", à une domination de la logique scientifique et économique sur le respect

\footnotetext{
1 A. GidDEnS, "Risiko, Vertrauen und Reflexivität", in U. BECK, A. GIDDENS, S. LASH, Reflexive Modernisierung. Eine Kontroverse, Suhrkamp, 1996, pp. 316337.

2 V. CAMPION-VINCENT, La légende des vols d'organes, Paris, Les Belles Lettres, 1997.
} 
du corps, à une transgression des valeurs primordiales associées à la vie et à la mort, à un déséquilibre entre le donneur -qui devient un pur objet- et le receveur -sujet de tous les soins. Des contre-discours de ce type manifestent peut-être la méfiance des journalistes/publics face à l'institution qui les exclue. En effet, les recherches soutenant la pratique des greffes se sont longtemps développées loin de l'espace public. De plus l'hôpital fait figure d' "autre monde" où l'on est admis comme objet de soins, non comme sujet de savoir. Les contrediscours naissent en réaction à une clôture qui sépare les institutions et la société, parce que les publics ne se sentent pas reconnus, pas compris par les institutions.

Face à ces réactions, quelles sont celles des acteurs engagés dans le domaine de la greffe ? Elles restent en fait classiques. En premier lieu, la mise en place en 1998 du registre des refus constitue le point de départ des campagnes d'information grand public. Ce fait est déjà intéressant en soi car plutôt que d'informer sur les modalités du refus, on sensibilise au don. Pour ce faire, des plaquettes visant à expliquer les greffes sont diffusées. On informe sur l'institution ("qui sont les hommes et les femmes de l'Établissement Français des Greffes ?", Que font-ils ? ...) et sur les aspects techniques et médicaux des greffes. Ainsi informée, voire même "éduquée", la personne devrait se prononcer "pour" le don d'organe.

La campagne de sensibilisation sur les greffes semble prendre pour modèle le don caritatif, dont les succès attestent l'efficacité. Toutefois une différence insurmontable semble compromettre l'efficacité du modèle caritatif lorsqu'il s'agit de don d'organes. Donner de l'argent, de la nourriture, des vêtements ou d'autres biens matériels revient à se séparer d'un surplus. On offre ce dont on n'a pas réellement besoin pour vivre. En revanche, le don du corps se conçoit difficilement comme un "partage" : le donneur ne garde rien, cède ce qu'il possède de plus intime, et ce n'est ni monnayable ni quantifiable. Bref, pour en revenir au premier point, il n'est peut-être pas "si normal" de donner ses organes.

Par ailleurs, l'institution tente de légitimer la transplantation en faisant appel à différents "garde-fous". L'inscription du champ dans les lois bioéthiques garantit la légalité de la pratique, interdit les abus. L'appel aux comités et représentants des religions monothéistes veut montrer qu'on prend le côté "humain" de la greffe en compte. Ainsi, un certain nombre de "ceintures de sécurité" sont déployées : elles montrent à tout un chacun que l'activité de la transplantation est 
transparente, éthique, légale, que tout écart à la règle est exclu. Bref, que les publics peuvent faire confiance et donc souscrire à la logique du don. Certes, mais cette "ceinture", si elle protège des abus, protège aussi la légitimité de l'institution : à aucun moment la logique de la greffe n'a été questionnée. Une fois que cette logique est inscrite dans le droit et l'éthique, les publics y ont de moins en moins accès, ainsi, il devient de plus en plus difficile de la questionner, d'en négocier les significations.

Peut-être nous trouvons nous dans une situation où il est moins question pour les publics de comprendre les logiques inhérentes à la transplantation, ses buts et ses possibilités, que de vouloir les comprendre. Si nous nous reportons à une notion proposée par le sociologue Ulrich Beck, il semblerait en effet que les publics se retranchent dans une attitude d'ignorance volontaire'. Il ne s'agit pas d'un déficit de savoir, mais une décision active de la part des acteurs. Le savoir scientifique et les décisions publiques qui en émanent peuvent paraître menaçants : ce type d'attitude vise alors à protéger des identités, des choix culturels ou individuels... En fait, il est difficile de critiquer ouvertement une institution qui sauve des vies et à laquelle on a souvent recours "pour soi". Les savoirs culturels et/ou personnels s'expriment alors en secret, ils sont repoussés dans l'intimité de la décision individuelle. Si nous suivons cette hypothèse, l'appel au don est peut-être ignoré parce que la logique biomédicale et la voix de l'institution ne font pas toujours sens pour les publics, parce qu'elles ne sont pas intégrables par les différents mondes sociaux. L'ignorance volontaire peut alors être comprise comme un "contre-pouvoir". Ce qui apparaîtrait alors, c'est la "formulation discursive implicite d'une relation de pouvoir entre soi et la science. Lorsque des individus réfléchissent (sur) leur ignorance des savoirs (scientifiques), ils dévoilent leur savoir sur l'ignorance et son rôle social"2.

1 U. BecK, World Risk Society, Cambridge, Polity Press, 1999, en particulier le chapitre 6 "Knowledge or Unawareness? Two Perspective on 'Reflexive Modernization"”, pp. 109-132.

2 M. MiCHEAL, "Ignoring science: discourses of ignorance in the public understanding of science", in B. WYNNE et al., Misunderstanding science, Cambridge, Cambridge University Press, p. 123. 